\title{
Individual and environmental contingencies associated with multiple suicide attempts among U.S. military personnel
}

Craig J. Bryan, PsyD, ABPP*a,b ${ }^{\text {a }}$ M. David Rudd, PhD, ABPP ${ }^{\mathrm{a}, \mathrm{c}}$, Evelyn Wertenberger, PhD, $\mathrm{LCSW}^{\mathrm{d}}$

${ }^{a}$ National Center for Veterans Studies, Salt Lake City, Utah

${ }^{b}$ The University of Utah, Department of Psychology, Salt Lake City, Utah

${ }^{c}$ The University of Memphis, Office of the President, Memphis, Tennessee

${ }^{\mathrm{d}}$ U.S. Army MEDDAC, Fort Carson, Colorado

*Corresponding Author: Craig J. Bryan, PsyD, ABPP, National Center for Veterans Studies, The University of Utah, 260 S. Central Campus Dr., Room 205, Salt Lake City, UT 84112, 801-5877978, craig.bryan@utah.edu 


\begin{abstract}
Suicidal behavior among U.S. military personnel persists as a significant public health issue. Previous research indicates the primary motive for suicide attempts among military personnel is the desire to reduce or alleviate emotional distress, a finding that converges with studies in nonmilitary samples. Much less is understood about the consequences of a first suicide attempt that could influence the occurrence of additional suicide attempts. In order to identify these contingencies, 134 active duty Soldiers who had attempted suicide ( $\mathrm{n}=69$ first-time attempters, $\mathrm{n}=65$ multiple attempters) participated in structured interviews focused on their experiences immediately following their first attempt. Soldiers were more likely to have made multiple suicide attempts if they were younger at the time of their first attempt, were not admitted to a hospital or treatment program after their first attempt, or experienced emotional and psychological relief immediately afterwards. Results suggest that Soldiers who experience emotional and/or psychological relief immediately after their first suicide attempt or do not receive treatment are more likely to make additional suicide attempts.
\end{abstract}

Key Words: military; suicide; emotion relief; functional model; reinforcement 


\section{Introduction}

Suicide among U.S. military personnel persists as a significant public health issue. Research indicates that many of the suicide risk factors identified in nonmilitary populations are applicable to the military. For example, psychiatric disorders such as depression and posttraumatic stress disorder (Ursano et al., 2015), life stressors such as relationship problems (Bryan et al., 2015a; Bryan and Rudd, 2012) and trauma exposure (Bryan et al., 2015b; Bryan et al., 2013a), and prior history of suicidal thoughts and behaviors (Bryan et al., 2014) have all been confirmed as risk factors among military personnel. Recent conceptual and empirical work suggest that the traditional risk factor approach for understanding suicidal behavior may be inadequate for understanding who will attempt suicide and why a suicide attempt will occur, however (Klonsky and May, 2014). Psychiatric diagnoses, for instance, are useful and robust predictors of suicidal thinking but are much less useful for determining which suicidal individuals will subsequently make a suicide attempt (Kessler et al., 1999). Even less is known about what factors motivate some suicide attempters to continue making multiple suicide attempts.

Research suggests that intentional self-injury and suicidal behaviors are largely motivated by the desire to reduce or avoid painful cognitive-affective states, although other motivations may be present as well (Bryan et al., 2013b; Nock and Prinstein, 2005). Based on the principles of learning theory and the functional model of intentional self-injury (Nock and Prinstein, 2005), multiple suicide attempts are most likely to occur among individuals who experience sufficient reinforcement following their first suicide attempts. Reinforcement can be understood on two dimensions, type of reinforcement (i.e., positive versus negative reinforcement) and source of reinforcement (i.e., internal/automatic versus external/social), which implies four general 
categories of motivation: automatic negative reinforcement, which refers to the desire to reduce or avoid unpleasant internal states such as depression, agitation, anger, or shame; automatic positive reinforcement, which refers to the desire to create or obtain a desirable internal state (e.g., calmness, relaxation, self-validation); social negative reinforcement, which refers to the desire to reduce or avoid unpleasant interpersonal situations or circumstances; and social positive reinforcement, which refers to the desire to obtain or create a desired environmental or interpersonal context.

Prior research indicates that automatic negative reinforcement is a primary motive for suicidal behavior among military personnel, being endorsed approximately twice as often as automatic positive reinforcement and both types of social reinforcement (Bryan et al., 2013b). In a sample of active duty Soldiers who had made a suicide attempt, for example, $100 \%$ of the suicide attempts were motivated by the desire "to stop bad feelings," and more than half indicated they were motivated by the desire to escape feelings of sadness, thoughts and memories, and negative feelings in general. Although automatic negative reinforcement motives were the most commonly reported, Soldiers often had multiple motives that cut across all four functional domains: $82 \%$ endorsed at least one social negative reinforcement motive (e.g., "to get away or escape from other people"), $80 \%$ endorsed at least one social positive reinforcement motive (e.g., "to communicate or let others know how desperate I was"), and 73\% endorsed at least one automatic positive reinforcement motive (e.g., "to punish myself"). Similar patterns have been observed in non-military samples including adolescent self-injurers (Nock and Prinstein, 2005) and women with borderline personality disorder (Brown et al., 2002). These findings align with various theoretical models that conceptualize suicide as a coping strategy for reducing or escaping from emotional pain (Joiner, 2005; Linehan, 1993; Rudd et al., 2004). 
The importance of automatic negative reinforcement further aligns with research indicating that individuals who have made two or more suicide attempts, often referred to as multiple attempters, report more severe levels of psychopathology (Forman et al., 2004; Rudd et al., 1996) and have relatively higher suicide risk than first-time suicide attempters (Bryan et al., 2015a; Joiner Jr and Rudd, 2000; Rudd et al., 1996). Although the exact reasons for this pattern are not yet clear, other lines of evidence indicate that multiple attempters experience a greater number of life stressors that tend to endure for longer periods of time (Bryan et al., 2015a; Joiner Jr and Rudd, 2000). Multiple attempters may therefore experience higher levels of emotional distress because they also experience a greater number of chronic stressors. Individuals who experience relief from aversive internal states and challenging life situations (i.e., negative reinforcement) and/or experience a positive internal state or a positive change in their life situation (i.e., positive reinforcement) after a first suicide attempt may therefore be vulnerable to adopting suicidal behavior as a long-term coping or problem-solving strategy.

Although useful for understanding the motives that drive suicidal behavior, research to date on the functional model has primarily focused on those motivations that existed before the individual made a suicide attempt (Brown et al., 2002; Bryan et al., 2013b; Nock and Prinstein, 2005). To our knowledge, no studies have examined the individual and social factors arising after a suicide attempt that may influence the likelihood that a person will attempt suicide again. The present study was therefore designed to fill this knowledge gap by examining the following questions in a clinical sample of active duty Army personnel:

1. Which reinforcement contingencies are experienced by military personnel following a first suicide attempt? 
2. Which reinforcement contingencies differentiate those military personnel who have made one suicide attempt from those who have made multiple suicide attempts?

Consistent with research supporting the importance of automatic negative reinforcement motives present before the engagement in nonsuicidal self-injury and suicide attempts, we hypothesized that automatic negative reinforcement contingencies experienced after a first suicide attempt would be significantly associated with repeat suicidal behavior.

\section{Method}

\subsection{Participants}

Participants were 134 active duty Soldiers accepted into a randomized clinical trial to prevent suicide attempts (Rudd et al., 2015). A detailed description of the parent study from which this sample was extracted, including a CONSORT chart, can be found in Rudd et al. (2015). In summary, a total of 206 Soldiers were invited to participate in the study, of which 176 (85.4\%) provided informed consent and 134 had a lifetime history of suicide attempt(s). For the current study, participants were required to meet the following inclusion criteria: (1) lifetime history of suicide attempt; (2) active duty military status; (3) age 18 or older; (4) ability to speak English; and (5) ability to understand and complete informed consent procedures. The only exclusion criterion was the presence of a medical or psychiatric condition that would preclude informed consent or participation in outpatient treatment (e.g., an active manic or psychotic episode). Of the 176 who provided informed consent, 134 (76.1\%) met all of the above inclusion criteria; 42 were excluded due to the absence of a lifetime history of suicide attempt.

Participants were predominantly male $(88.8 \%)$ and ranged in age from 19 to 44 years $(\mathrm{M}=26.94, \mathrm{SD}=5.96)$. Self-reported racial distribution was $70.1 \%$ Caucasian, $11.9 \%$ AfricanAmerican, 2.2\% Asian, 2.2\% Pacific Islander, 4.5\% Native American, and 9.0\% other. Hispanic 
ethnicity, assessed separate from race, was endorsed by $24.6 \%$ of participants. Rank distribution was $70.9 \%$ junior enlisted (E1-E4), 25.4\% noncommissioned officer (E5-E6), and 3.7\% senior noncommissioned officer (E7-E9). Participants had served in the military for 0 to 22 years $(\mathrm{M}=5.49, \mathrm{SD}=4.04)$ and had deployed a total of 0 to 8 times $(\mathrm{M}=1.63, \mathrm{SD}=1.35)$.

\subsection{Instruments}

All assessments were completed prior to treatment assignment. History of suicide attempts and the characteristics of each attempt (i.e., medical lethality, suicidal intent, method) were assessed using the Suicide Attempt Self Injury Interview (SASII; Linehan et al., 2006a). Medical lethality is scored using a 6-point scale ranging from 1 ("very low"; e.g., taking up to 5 pills, scratching, reopening wounds) to 6 ("severe"; e.g., pulling trigger of loaded gun aimed at vital area, jumping from height $>20$ feet, hanging with feet above the ground). Suicidal intent is scored using a 7-point scale that asks patients if they were thinking about suicide or wishing they were dead just before or at the time of their behavior. Response options range from 0 ("not at all") to 6 ("I was extremely serious, intended to die, and was not ambivalent at all"). Behavioral contingencies experienced after participants' first suicide attempts were assessed with a 29-item list of potential consequences that was derived from open-ended interviews with patients who had attempted suicide and/or engaged in nonsuicidal self-injury (Brown et al., 2002). Consistent with standardized SASII administration procedures, participants were directed to indicate which of the events or experiences happened to them immediately following the suicide attempt. The validity of the SASII items has been established and interrater reliabilities exceed .87 (Linehan et al., 2006a). Furthermore, the validity of suicide attempt classification based on retrospective report compared to other information sources (i.e., therapist notes, weekly patient self-report, 
medical records) ranges between 75 and $91 \%$ agreement. The interrater reliability in the present study was excellent $(\kappa=0.96)$.

Lifetime history of major depressive disorder, posttraumatic stress disorder, substance use disorder, and borderline personality disorder was established using the Structured Clinical Interview for DSM-IV for both Axis I (SCID-I; First et al., 2012) and Axis II (SCID-II; First et al., 1997) disorders.

\subsection{Procedures}

Upon referral, the evaluator used the SASII to assess the characteristics of the first suicide attempt for all participants. For those with multiple suicide attempts, characteristics of the most recent suicide attempt and, where applicable, a third suicide attempt were also assessed. Only two (1.5\%) participants had more than three lifetime suicide attempts (one had a total of four suicide attempts and one had five suicide attempts). Out of a total of 229 lifetime suicide attempts, $226(98.7 \%)$ were assessed in the present study.

\subsection{Data Analysis}

To test the associations of post-attempt behavioral contingencies with suicide attempt status (i.e., first-time versus multiple suicide attempts), we used univariate and multivariate logistic regression with age of first suicide attempt and psychiatric diagnoses entered as covariates. Firth's penalized likelihood approach was used for all logistic regression analyses to address issues of separability and small sample size (Heinze, 2006). In addition to calculating unadjusted p-values, we calculated confidence intervals for all effect size statistics. A previous report (Bryan and Rudd, 2015) utilizing this same sample found no differences between firsttime and multiple attempters on any demographic variable; these variables therefore were not included as covariates. Raw two-sided $\mathrm{p}$ values are presented unadjusted for multiple 
comparisons so that adjustment of choice (e.g., Bonferroni, sequential Bonferroni, Sidak) can be performed by the reader.

\section{Results}

Of the 134 participants, $69(51.1 \%)$ were first-time attempters and $65(58.5 \%)$ were multiple attempters. Participants were assessed a median of 46 days after the most recent suicide attempt; $39.3 \%$ were assessed 0-30 days after, $26.9 \%$ were assessed 31-180 days after, 10.4\% were assessed 181-365 days after, and 23.4\% were assessed more than one year after the most recent suicide attempt.

There were no differences between first-time and multiple attempters in terms of gender or age at the time of enrollment (see Table 1). Multiple attempters were significantly younger than first-time attempters when they made their first suicide attempt however: $M=18.66$, $\mathrm{SD}=6.87$ vs. $\mathrm{M}=24.30, \mathrm{SD}=6.89, \mathrm{t}(132)=4.75, \mathrm{p}<.001$. The primary methods used during participants' first suicide attempt included medication/drug overdose (40.3\%), scratching/cutting (19.4\%), firearm (14.9\%), hanging (8.2\%), jumping from a height (4.5\%), alcohol poisoning $(3.0 \%)$, transportation-related $(3.0 \%)$, stepping into traffic $(1.5 \%)$, self-poisoning or caustic substance $(1.5 \%)$, and other $(3.7 \%)$; there were no differences between first-time and multiple attempters in terms of method used for their first suicide attempt. The medical lethality $(t(132)=0.13, p=.894)$ and intensity of subjective suicidal intent associated with the first suicide attempt $(\mathrm{t}(132)=0.14, \mathrm{p}=.887)$ also did not differ between first-time and multiple attempters.

We first computed the associations of the 29 behavioral contingencies with first-time versus multiple attempter status; these are reported as proportions in Table 2. Three contingencies differentiated first-time from multiple attempters. Multiple attempters were less likely to report gaining admission into a hospital or treatment program (21.5\% vs. $39.1 \%$; 
$\mathrm{OR}=0.43[0.20,0.92], \mathrm{p}=.027)$ but were more likely to feel that other people treated them better $(18.5 \%$ vs. $7.2 \% ; \mathrm{OR}=2.90[0.96,8.75], \mathrm{p}=.051)$ and to feel that they experienced relief from a terrible state of mind (24.6\% vs. $11.6 \%$; OR=2.49 [1.00, 6.30], $\mathrm{p}=.049)$. Results indicated that multiple attempters were significantly more likely to have been younger when they first attempted suicide, $\mathrm{OR}=.88[.82, .96], \mathrm{p}=.002$, less likely to have gained admission to a hospital or treatment facility after the attempt, $\mathrm{OR}=.34[.14, .80], \mathrm{p}=.013$, and more likely to have experienced relief from a terrible state of mind after the attempt, $O R=3.21[1.10,9.39], \mathrm{p}=.033$.

To test the sensitivity of these findings while controlling for other relevant variables, two multivariate logistic regression models were constructed. In the first model, all three contingencies were entered simultaneously along with several other variables associated with the first suicide attempt: medical lethality, intensity of suicidal intent, and age of first attempt. In the second model, lifetime history of psychiatric diagnosis, gender, and current age were entered as covariates. In both models, gaining admission to a hospital or treatment facility remained associated with significantly decreased risk for multiple attempts whereas experiencing relief from a terrible state of mind was associated with significantly increased risk for multiple attempts (see Table 3).

A follow-up analysis was conducted by rationally clustering the 29 contingencies into the four superordinate functions, consistent with previous research (Brown et al., 2002; Bryan et al., 2013c). Similar to Bryan et al. (2013c), the mean number of endorsed contingencies per function was calculated to determine the relative weight of each function relative to the others. Mean scores are reported in Table 4. The four clusters were entered into a multivariate logistic regression model with all of the previously-mentioned covariates. The only cluster that 
significantly differentiated single and multiple attempters was automatic negative reinforcement $(\mathrm{AOR}=10.37[1.03,104.30], \mathrm{p}=.047)$

\section{Discussion}

Consistent with the automatic negative reinforcement contingency described by the functional model of self-injury (Nock and Prinstein, 2005), existing research indicates that the desire to alleviate or reduce emotional pain is the primary motive that drives individuals, including military personnel, to attempt suicide (Brown et al., 2002; Bryan et al., 2013b). Much less is known about how the reinforcement contingencies that follow suicide attempts might influence whether or not a suicide attempter will make another suicide attempt again in the future. Results of the current study suggest that two consequences in particular, receipt of treatment and reduction of emotional distress, may contribute to repeat suicide attempts when experienced immediately after a first suicide attempt. Follow-up analyses further indicated that, of all four reinforcement contingencies, automatic negative reinforcement (i.e., emotion relief) may be an especially important domain. To our knowledge, this is the first empirical investigation of the behavioral contingencies that may reinforce and sustain multiple suicide attempts.

In the current study, multiple attempters were approximately six years younger when they made their first suicide attempt as compared to first-time attempters, suggesting that multiple attempters first engage in suicidal behavior much earlier in life. This is consistent with the fluid vulnerability theory of suicide (Rudd, 2006), which posits that multiple attempters have predisposing cognitive, affective, behavioral, and physiological/genetic vulnerabilities that increase their propensity to experience suicidal crises in response to triggering events. Because these vulnerabilities develop very early in the lifespan, suicidal behavior is more likely to emerge 
at a much younger age. No other characteristic of the first suicide attempt examined in this study — method used, medical severity of the attempt, or intensity of suicidal intent—was associated with multiple suicide attempt status. Likewise, lifetime history of psychiatric disorder did not differentiate between first-time and multiple attempters, a finding that aligns with previous research (Bryan and Rudd, 2015; Rudd et al., 1996).

In contrast to these findings, differences between first-time and multiple attempters were observed with respect to the behavioral contingencies the experienced following a first suicide attempt. First, consistent with expectations, multiple attempters were more likely to report experiencing relief from a terrible state of mind, a finding that aligns with automatic negative reinforcement. The emotional relief reported by multiple attempters converges with prior research indicating that emotion relief is also the primary motive that drives suicide attempts among military personnel (Bryan et al., 2013b). Repeated suicidal behavior among military personnel might therefore occur in part because, to them, it has proven to be an effective coping strategy.

Multiple attempters were also more likely than first-time attempters to report that they felt better treated by others following their first suicide attempt. This finding suggests that service members who are vulnerable to making repeated suicide attempts are more likely to perceive a positive change in their interpersonal relations following a first suicide attempt. This should not be misconstrued to suggest that service members who make suicide attempts should be treated poorly, however. It is possible, for instance, that participants felt that they were being poorly treated before the suicide attempt, but this poor treatment reduced after the suicide attempt occurred. Another possibility is that participants perceived a shift in others' actions even 
when no such shift occurred. Additional research focused on the interpersonal dynamics following a first suicide attempt is needed to further clarify this finding.

Finally, multiple attempters were much less likely than first-time attempters to have gained admission to a hospital or treatment program after their first suicide attempt. With respect to gaining access to hospital or treatment programs, randomized clinical trials indicate that certain treatments such as dialectical behavior therapy (Linehan et al., 1991; Linehan et al., 2006b) and brief cognitive behavioral therapies (Brown et al., 2005; Rudd et al., 2015) contribute to significantly reduced rates of repeat suicide attempts among suicide attempters. Unfortunately, we were unable to determine which treatments were received by participants following their first suicide attempts.

Taken together, these results suggest that participants who felt better in some capacity following a first suicide attempt were more likely to have made another suicide attempt whereas those who received treatment soon after making their first suicide attempt were less likely to have made another suicide attempt. The present study has several limitations that warrant discussion when drawing conclusion. First, data were based on retrospective self-report methods, which could introduce recall bias. Although previous research supports the reliability of the methods used in our study (Linehan et al., 2006a), retrospective recall of what one experienced following a suicide attempt that occurred in the past might nonetheless be influenced by the passage of time. Prospective studies that assess the motivations that drive suicidal behavior prior to a suicide attempt as well as the consequences that are experienced immediately after a suicide attempt are needed to confirm these results. Future research that clarifies how the match (or mismatch) between an individual's expectations and motives prior to a suicide attempt and experiences after a suicide attempt might reinforce additional suicidal behavior would also be 
helpful. Related to this, it is possible that some of the first-time attempters in our sample will make a second suicide attempt at a later date. Additional studies are therefore need to determine if certain post-attempt contingencies can prospectively predict repeat suicidal behavior, both fatal and nonfatal. Second, the present sample was limited to active duty Soldiers who voluntarily presented to an outpatient mental health clinic for treatment; results therefore may not generalize to nonclinical samples or nonmilitary samples. Finally, our sample was predominantly male, which limits the possibility to consider potential gender differences. Although the gender distribution observed in this study is comparable to that of the military more broadly, conclusions about the applicability of findings to female military personnel should be made with caution until studies with large numbers of female participants are completed. Despite these limitations, these results provide clinically useful information about how the individual and environmental consequences of suicidal behavior may influence some military personnel who have attempted suicide to make additional suicide attempts. 


\section{Acknowledgements}

This project was supported in part through research funding by the Department of Defense award \#W81XWH-09-1-0569 (M. David Rudd, Principal Investigator). The funding agency had no involvement in the study design, data collection, analysis, or manuscript preparation. The views expressed in this article are those of the authors and do not necessarily represent the official position or policy of the U.S. Government, the Department of Defense, or the U.S. Army. 


\section{References}

Brown, G.K., Ten Have, T., Henriques, G.R., Xie, S.X., Hollander, J.E., Beck, A.T., 2005. Cognitive therapy for the prevention of suicide attempts: a randomized controlled trial. JAMA 294 (5), 563-570.

Brown, M.Z., Comtois, K.A., Linehan, M.M., 2002. Reasons for suicide attempts and nonsuicidal self-injury in women with borderline personality disorder. Journal of Abnormal Psychology 111 (1), 198-202.

Bryan, C.J., Bryan, A.O., Ray-Sannerud, B.N., Etienne, N., Morrow, C.E., 2014. Suicide attempts before joining the military increase risk for suicide attempts and severity of suicidal ideation among military personnel and veterans. Comprehensive Psychiatry 55 (3), 534-541.

Bryan, C.J., Clemans, T.A., Leeson, B., Rudd, M.D., 2015a. Acute vs. Chronic Stressors, Multiple Suicide Attempts, and Persistent Suicide Ideation in US Soldiers. The Journal of nervous and mental disease 203 (1), 48-53 10.1097/NMD.0000000000000236.

Bryan, C.J., Griffith, J., Pace, B.T., Hinkson, K., Bryan, A.O., Clemans, T., Imel, Z., 2015 b. Combat exposure and risk for suicidal thoughts and behaviors among military personnel and veterans: a systematic review and meta-analysis. Suicide and Life-Threatening Behavior.

Bryan, C.J., McNaugton-Cassill, M., Osman, A., Hernandez, A.M., 2013a. The associations of physical and sexual assault with suicide risk in nonclinical military and undergraduate samples. Suicide and Life-Threatening Behavior 43 (2), 223-234.

Bryan, C.J., Rudd, M.D., 2012. Life stressors, emotional distress, and trauma-related thoughts occurring in the $24 \mathrm{~h}$ preceding active duty U.S. soldiers' suicide attempts. Journal of Psychiatric Research 46 (7), 843-848.

Bryan, C.J., Rudd, M.D., 2015. Demographic and Diagnostic Differences Among Suicide Ideators, Single Attempters, and Multiple Attempters Among Military Personnel and Veterans Receiving Outpatient Mental Health Care. Military Behavioral Health 3 (4), 289-295.

Bryan, C.J., Rudd, M.D., Wertenberger, E., 2013b. Reasons for suicide attempts in a clinical sample of active duty soldiers. Journal of Affective Disorders 144 (1-2), 148-152.

Bryan, C.J., Rudd, M.D., Wertenberger, E., 2013c. Reasons for suicide attempts in a clinical sample of active duty soldiers. J Affect Disord 144 (1), 148-152.

First, M.B., Spitzer, R.L., Gibbon, M., Williams, J.B., 2012. Structured Clinical Interview for DSM-IV® Axis I Disorders (SCID-I), Clinician Version, Administration Booklet. American Psychiatric Pub.

First, M.B., Spitzer, R.L., Gibbon, M., Williams, J.B.W., 1997. Structured Clinical Interview for DSM-IV Personality Disorders (SCID-II). American Psychiatric Press, Washington, DC.

Forman, E.M., Berk, M.S., Henriques, G.R., Brown, G.K., Beck, A.T., 2004. History of Multiple Suicide Attempts as a Behavioral Marker of Severe Psychopathology. American Journal of Psychiatry 161 (3), 437-443.

Heinze, G., 2006. A comparative investigation of methods for logistic regression with separated or nearly separated data. Statistics in medicine 25 (24), 4216-4226.

Joiner Jr, T.E., Rudd, M.D., 2000. Intensity and duration of suicidal crisis vary as a function of previous suicide attempts and negative life events. Journal of Consulting and Clinical Psychology 68 (5), 909-916. 
Joiner, T., 2005. Why people die by suicide. Harvard University Press.

Kessler, R.C., Borges, G., Walters, E.E., 1999. Prevalence of and risk factors for lifetime suicide attempts in the National Comorbidity Survey. Archives of General Psychiatry 56 (7), 617-626.

Klonsky, E.D., May, A.M., 2014. Differentiating Suicide Attempters from Suicide Ideators: A Critical Frontier for Suicidology Research. Suicide and Life-Threatening Behavior 44 (1), $1-5$.

Linehan, M., 1993. Cognitive-behavioral treatment of borderline personality disorder. Guilford Press, New York, NY.

Linehan, M.M., Armstrong, H.E., Suarez, A., Allmon, D., Heard, H.L., 1991. Cognitivebehavioral treatment of chronically parasuicidal borderline patients. Archives of General Psychiatry 48 (12), 1060-1064.

Linehan, M.M., Comtois, K.A., Brown, M.Z., Heard, H.L., Wagner, A., 2006a. Suicide Attempt Self-Injury Interview (SASII): development, reliability, and validity of a scale to assess suicide attempts and intentional self-injury. Psychological Assessment 18 (3), 303-312.

Linehan, M.M., Comtois, K.A., Murray, A.M., Brown, M.Z., Gallop, R.J., Heard, H.L., Korslund, K.E., Tutek, D.A., Reynolds, S.K., Lindenboim, N., 2006b. Two-year randomized controlled trial and follow-up of dialectical behavior therapy vs therapy by experts for suicidal behaviors and borderline personality disorder. Archives of General Psychiatry 63 (7), 757-766.

Nock, M.K., Prinstein, M.J., 2005. Contextual features and behavioral functions of selfmutilation among adolescents. Journal of Abnormal Psychology 114 (1), 140.

Rudd, M.D., 2006. Fluid vulnerability theory: a cognitive approach to understanding the process of acute and chronic risk., in: Ellis, T.E. (Ed.), Cognition and Suicide: Theory, Research, and Therapy. American Psychological Association, Washington, DC, pp. 355-368.

Rudd, M.D., Bryan, C.J., Wertenberger, E., Peterson, A.L., Young-McCaughon, S., Mintz, J., Williams, S.R., Arne, K.A., Breitbach, J., Delano, K.A., Wilkinson, E., Bruce, T.O., 2015. Brief cognitive behavioral therapy effects on post-treatment suicide attempts in a military sample: results of a 2-year randomized clinical trial. American Journal of Psychiatry.

Rudd, M.D., Joiner, T., Rajab, M.H., 1996. Relationships among suicide ideators, attempters, and multiple attempters in a young-adult sample. Journal of Abnormal Psychology 105 (4), 541-550.

Rudd, M.D., Joiner, T.E., Rajab, M.H., 2004. Treating suicidal behavior: An effective, timelimited approach. Guilford Press.

Ursano, R.J., Heeringa, S.G., Stein, M.B., Jain, S., Raman, R., Sun, X., Chiu, W.T., Colpe, L.J., Fullerton, C.S., Gilman, S.E., Hwang, I., Naifeh, J.A., Nock, M.K., Rosellini, A.J., Sampson, N.A., Schoenbaum, M., Zaslavsky, A.M., Kessler, R.C., 2015. Prevalence and correlates of suicidal behavior among new soldiers in the u.s. Army: results from the army study to assess risk and resilience in servicemembers (army starrs). Depression and Anxiety 32 (1), 3-12. 
Table 1

Differences between single and multiple suicide attempters in a clinical sample of active duty Soldiers

\begin{tabular}{lcc}
\hline & $\begin{array}{c}\text { Single } \\
(\mathrm{n}=69)\end{array}$ & $\begin{array}{c}\text { Multiple } \\
(\mathrm{n}=65)\end{array}$ \\
\hline Age & $26.99(6.02)$ & $26.89(5.94)$ \\
Female & $7(10.1 \%)$ & $7(10.8 \%)$ \\
Characteristics of first attempt & & \\
$\quad$ Age & $24.30(6.89)$ & $18.66(6.87)$ \\
Medical lethality & $3.93(1.78)$ & $3.97(1.83)$ \\
$\quad$ Intensity of suicidal intent & $4.84(1.45)$ & $4.88(1.52)$ \\
Lifetime DSM-IV diagnoses & & \\
$\quad$ Major depressive disorder & $50(72.5 \%)$ & $51(78.5 \%)$ \\
$\quad$ Substance use disorder & $2(2.9 \%)$ & $2(3.1 \%)$ \\
$\quad$ Posttraumatic stress disorder & $26(37.7 \%)$ & $25(38.5 \%)$ \\
Borderline personality disorder & $4(5.8 \%)$ & $10(15.4 \%)$ \\
\hline
\end{tabular}




\section{Table 2}

\section{Frequency of endorsement of each behavioral contingency following a first suicide attempt among single and multiple suicide attempters}

\begin{tabular}{|c|c|c|c|c|c|c|c|}
\hline \multirow[b]{2}{*}{ Behavioral contingency } & \multicolumn{2}{|c|}{ Single } & \multicolumn{2}{|c|}{ Multiple } & \multirow[b]{2}{*}{ OR } & \multirow[b]{2}{*}[95\%\mathrm{CI}]{} & \multirow[b]{2}{*}{$\mathrm{p}$} \\
\hline & $\mathrm{n}$ & $\%$ & $\mathrm{n}$ & $\%$ & & & \\
\hline 1. Bad feelings stopped & 8 & 11.6 & 9 & 13.8 & 1.23 & {$[0.44,3.40]$} & 0.695 \\
\hline 2. Others understood how desperate you are/were & 15 & 21.7 & 12 & 18.5 & 0.82 & {$[0.35,1.90]$} & 0.636 \\
\hline 3. You got help & 32 & 46.4 & 21 & 32.3 & 0.55 & {$[0.27,1.11]$} & 0.096 \\
\hline 4. You gained admission into a hospital or treatment program & 27 & 39.1 & 14 & 21.5 & 0.43 & {$[0.20,0.92]$} & 0.027 \\
\hline 5. You felt something, even if it was pain & 15 & 21.7 & 19 & 29.2 & 1.49 & {$[0.68,3.25]$} & 0.319 \\
\hline 6. You felt punished or succeeded in punishing yourself & 4 & 5.8 & 7 & 10.8 & 1.96 & {$[0.55,7.04]$} & 0.295 \\
\hline 7. You got a vacation from having to try so hard & 4 & 5.8 & 2 & 3.1 & 0.52 & {$[0.09,2.92]$} & 0.447 \\
\hline 8. You got out of doing something & 2 & 2.9 & 1 & 1.5 & 0.52 & {$[0.05,5.91]$} & 0.595 \\
\hline 9. You shocked or impressed others & 11 & 15.9 & 6 & 9.2 & 0.54 & {$[0.19,1.55]$} & 0.243 \\
\hline 10. You proved to yourself that things really were bad & 12 & 17.4 & 12 & 18.5 & 1.08 & {$[0.44,2.60]$} & 0.872 \\
\hline 11. It gave you something, anything to do & 3 & 4.3 & 0 & 0.0 & 0.15 & {$[0.01,2.86]$} & 0.089 \\
\hline 12. Other people treated you better & 5 & 7.2 & 12 & 18.5 & 2.90 & {$[0.96,8.75]$} & 0.051 \\
\hline 13. You got back at or hurt someone & 0 & 0.0 & 5 & 7.7 & 12.64 & {$[0.69,233.24]$} & 0.119 \\
\hline 14. Other people were better off than before you harmed yourself & 3 & 4.3 & 2 & 3.1 & 0.70 & {$[0.11,4.32]$} & 0.698 \\
\hline 15. You got away or escaped & 7 & 10.1 & 12 & 18.5 & 2.01 & {$[0.74,5.46]$} & 0.168 \\
\hline 16. You stopped feeling numb or dead & 7 & 10.1 & 11 & 16.9 & 1.80 & {$[0.65,4.98]$} & 0.250 \\
\hline 17. You prevented yourself from being hurt in a worse way & 7 & 10.1 & 4 & 6.2 & 0.58 & {$[0.16,2.09]$} & 0.400 \\
\hline 18. Feelings of anger, frustration, or rage stopped & 8 & 11.6 & 12 & 18.5 & 1.73 & {$[0.66,4.54]$} & 0.265 \\
\hline 19. Others realized how wrong they are/were & 3 & 4.3 & 3 & 4.6 & 1.06 & {$[0.21,5.47]$} & 0.940 \\
\hline 20. Feelings of anxiety or terror stopped & 4 & 5.8 & 9 & 13.8 & 2.61 & {$[0.77,8.94]$} & 0.116 \\
\hline 21. You were distracted from other problems & 11 & 15.9 & 13 & 20.0 & 1.32 & [0.54. 3.20] & 0.540 \\
\hline 22. Feelings of aloneness, emptiness, or isolation stopped & 7 & 10.1 & 9 & 13.8 & 1.42 & {$[0.50,4.07]$} & 0.509 \\
\hline 23. Feelings of self-hatred/shame stopped & 5 & 7.2 & 5 & 7.7 & 1.07 & {$[0.29,3.87]$} & 0.922 \\
\hline $\begin{array}{l}\text { 24. Your (self-injury/suicide attempt/overdose) expressed your anger or } \\
\text { frustration }\end{array}$ & 16 & 23.2 & 16 & 24.6 & 1.08 & {$[0.49,2.39]$} & 0.846 \\
\hline 25. You experienced relief from a terrible state of mind & 8 & 11.6 & 16 & 24.6 & 2.49 & {$[1.00,6.30]$} & 0.049 \\
\hline 26. Feelings of sadness stopped & 4 & 5.8 & 8 & 12.3 & 2.28 & {$[0.65,7.97]$} & 0.187 \\
\hline $\begin{array}{l}\text { 27. You stopped feeling empty inside, as if you were unreal, or } \\
\text { disconnected from your feelings }\end{array}$ & 4 & 5.8 & 8 & 12.3 & 2.28 & {$[0.65,7.97]$} & 0.187 \\
\hline 28. Feelings of depression stopped & 4 & 5.8 & 9 & 13.8 & 2.61 & {$[0.76,8.94]$} & 0.116 \\
\hline 29. You felt worse about yourself or felt more self-hatred/shame & 29 & 42.0 & 19 & 29.2 & 0.57 & {$[0.28,1.17]$} & 0.123 \\
\hline
\end{tabular}


Reinforcing suicide attempts 20

\section{Table 3}

Results of multivariate logistic regressions predicting multiple (vs. single) suicide attempts

\begin{tabular}{lllllll}
\hline Predictor & OR & {$[95 \% \mathrm{CI}]$} & $\mathrm{p}$ & $\mathrm{OR}$ & {$[95 \% \mathrm{CI}]$} & $\mathrm{p}$ \\
\hline Features of first attempt & & & & & & \\
$\quad$ Medical lethality & 1.10 & {$[0.88,1.37]$} & 0.404 & 1.14 & {$[0.91,1.43]$} & 0.258 \\
$\quad$ Intensity of suicidal intent & 0.99 & {$[0.76,1.28]$} & 0.934 & 0.99 & {$[0.76,1.30]$} & 0.964 \\
$\quad$ Age of first attempt & 0.88 & {$[0.82,0.96]$} & 0.002 & 0.84 & {$[0.76,0.93]$} & 0.001 \\
Contingencies following first attempt & & & & & & \\
$\quad$ Gained admission to hospital or treatment & 0.34 & {$[0.14,0.80]$} & 0.013 & 0.37 & {$[0.16,0.90]$} & 0.027 \\
$\quad$ Other people treated you better & 2.32 & {$[0.74,7.29]$} & 0.150 & 2.25 & {$[0.64,7.85]$} & 0.205 \\
$\quad$ Experienced relief from terrible state of mind & 3.21 & {$[1.10,9.39]$} & 0.033 & 3.10 & {$[1.03,9.36]$} & 0.045 \\
Covariates & & & & & & \\
$\quad$ Major depressive disorder & -- & -- & -- & 1.11 & {$[0.46,2.66]$} & 0.817 \\
$\quad$ Substance use disorder & -- & -- & -- & 1.38 & {$[0.15,12.83]$} & 0.778 \\
$\quad$ Posttraumatic stress disorder & -- & -- & -- & 0.99 & {$[0.40,2.47]$} & 0.985 \\
$\quad$ Borderline personality disorder & -- & -- & -- & 1.27 & {$[0.23,7.11]$} & 0.789 \\
Age & -- & -- & -- & 1.12 & {$[1.00,1.25]$} & 0.059 \\
$\quad$ Gender & -- & -- & -- & 0.88 & {$[0.21,3.81]$} & 0.869 \\
\hline
\end{tabular}




\section{Table 4}

Mean contingency cluster scores (with standard deviations) for multiple and single suicide attempters

\begin{tabular}{lcc}
\hline Function & $\begin{array}{c}\text { Single } \\
\text { Attempter } \\
(\mathrm{n}=69)\end{array}$ & $\begin{array}{c}\text { Multiple } \\
\text { Attempter } \\
(\mathrm{n}=65)\end{array}$ \\
\hline A-NR & $0.09(0.19)$ & $0.15(0.22)$ \\
A-PR & $0.11(0.16)$ & $0.14(0.16)$ \\
S-NR & $0.09(0.19)$ & $0.10(0.15)$ \\
S-PR & $0.18(0.16)$ & $0.16(0.17)$ \\
\hline
\end{tabular}

A-NR = automatic negative reinforcement; A-PR = automatic positive reinforcement; S-NR = social negative reinforcement; $\mathrm{S}-\mathrm{PR}=$ social positive reinforcement. 